\title{
Diplopia from abducens nerve paresis as a presenting symptom of COVID-19: a case report and review of literature
}

Geulah Sarah Ben-David ( $\sim$ gbendavi@gmail.com )

Emek Medical Center

Orly Halachmi-Eyal

Emek Medical Center

Hana Shyriaiev

Emek Medical Center

Shay Brikman

Emek Medical Center

Guy Dori

Emek Medical Center

Daniel Briscoe

Emek Medical Center

Brief report

Keywords: novel coronavirus, COVID-19, diplopia, ophthalmoparesis

Posted Date: August 5th, 2020

DOI: https://doi.org/10.21203/rs.3.rs-53271/v1

License: (1) This work is licensed under a Creative Commons Attribution 4.0 International License.

Read Full License 


\section{Abstract}

Neurological manifestations of COVID-19 are not well understood. We report a case of a 44-year-old man who presented with fever, double vision, and headache and was subsequently diagnosed with COVID-19 and transient abducens nerve paresis. He did not present with any respiratory symptoms or additional specific neurological findings. We suggest that as the number of cases rises worldwide, physicians should have a greater index of suspicion for COVID-19 in patients with cranial neuropathies even in mild cases without typical respiratory symptoms.

\section{Introduction}

The novel coronavirus disease (COVID-19) originating in Wuhan, China has become a widespread global pandemic with over 16 million cases and over 650,000 deaths worldwide. ${ }^{1}$ Linked to the family of severe acute respiratory syndrome coronavirus (SARS-CoV), the new coronavirus is designated SARS-CoV-2 with typical symptoms including fever, cough, and shortness of breath. ${ }^{1}$ The main ocular abnormality that has been reported is conjunctivitis. Recently, in a study of 38 patients with confirmed COVID-19, 12 (31.6\%) had conjunctivitis. ${ }^{2}$ Neurological manifestations are reported in 78/214 (36.4\%) of COVID-19 patients including central nervous system (e.g., headache) and peripheral nervous system (e.g., taste, smell, and vision impairment) manifestations. ${ }^{3}$ Diplopia secondary to abducens nerve palsy in adults is often secondary to vascular disease, trauma, tumors, autoimmune, inflammatory, and idiopathic causes. Spontaneous recovery may occur in unilateral, isolated, and benign cases. ${ }^{4}$ Diplopia secondary to cranial nerve motor neuropathies may be associated with viral etiology, however, it is not a common presenting symptom of COVID-19. Awareness of cranial nerve involvement may increase the index of suspicion for diagnosing patients with COVID-19 and potentially prevent disease transmission. Here, we describe the first case of isolated abducens paresis and associated diplopia as the sole ocular abnormality in a generally healthy patient with COVID-19.

\section{Case Report}

A 44-year-old generally healthy man referred for ophthalmologic consultation in a hospitalized institutional setting presented with a 5-day history of fever, double vision, and headache. The patient also noted fatigue, generalized muscle weakness, and loss of appetite for one week. Several days prior to admission, he visited a healthcare practitioner and was sent home with antipyretic treatment. Due to lack of respiratory symptoms, COVID-19 was not suspected initially. The patient had no known medical or ocular history, medications, or allergies. As his symptoms did not resolve, he was admitted to the hospital without any respiratory symptoms. Blood tests demonstrated mild lymphopenia $\left(1.28 \times 10^{9} / \mathrm{L}\right)$, elevated C-reactive protein $(92 \mathrm{mg} / \mathrm{L})$, and D-dimer level $(1.3 \mathrm{ug} / \mathrm{ml})$ consistent with COVID-19. Lung auscultation and chest $\mathrm{x}$-ray indicated a bilateral pneumonia-like illness. Computed tomography of the head showed no pathological findings. Reverse transcriptase-polymerase chain reaction (RT-PCR) for SARS-CoV-2 was detected in the patient's first nasopharyngeal swab Ophthalmologic examination, performed with 
complete personal protective equipment, revealed binocular diplopia and a limitation to abduction in the left eye (Fig. 1). Orthophoria was noted in right gaze, and there was no additional cranial nerve involvement. Ophthalmologic examination including pupillary response, anterior pole, and dilated fundus exam was otherwise unremarkable. Neurological examination was normal and did not show evidence of meningeal irritation. Lumbar puncture and magnetic resonance imaging were considered but not performed due to resolution of symptoms within 5 days of admission. Treatment included azithromycin and hydroxychloroquine for 5 days as was according to the local infectious disease protocol. The patient was discharged to state-mandatory quarantine, and ten days later he had improved and was negative for SARS-CoV-2.

\section{Discussion}

The most plausible diagnosis in our patient without any prior medical or ocular history was isolated abducens paresis associated with COVID-19 infection. Vascular, neoplastic, and traumatic etiologies as well as pre-existing strabismus, thyroid eye disease, and myasthenia gravis were ruled out. A possible mechanism of the viral etiology in the current case is direct invasion of the abducens nerve. Prior CoV studies have described viral spread via the olfactory nerves possibly due a viral interaction with the membrane bound angiotensin-converting enzyme 2 receptor. ${ }^{5}$ Another hypothesized mechanism is hyperactivation of monocytes and dysregulated macrophages leading to a hyperinflammatory immune response. ${ }^{6}$ Given that the diplopia occurred at the beginning of our patient's disease course, direct viral spread was suspected although an immune response to viral infection could not be ruled out.

Recently, diplopia has been suggested as a symptom of COVID-19 linked to ophthalmoparesis and Miller Fisher Syndrome, a demyelinating inflammatory polyneuropathy. Across three recent studies, one patient presented with partial third nerve palsy and accompanying bilateral sixth nerve palsy, one with complete third nerve palsy only, one with bilateral sixth nerve palsy, and two with unilateral sixth nerve palsy. $7,8,9$ Similar to the two aforementioned cases, the precise mechanism in our case remains unknown. In these previously mentioned studies, patients who presented with third nerve palsy with or without additional sixth nerve palsy demonstrated a more severe manifestation of their infection and required more intensive, in-patient treatment. Whether we can provide a clinical guideline for future cases remains undetermined. However, the presentation and management of isolated ocular motor cranial nerve paresis, as in our case, may be associated with a less complicated disease process whereas patients who present with persistent sixth nerve palsy or additional third nerve palsy may be correlated with greater disease severity.

Since neuroimaging and cerebral spinal fluid analysis were not acquired for our patient who presented during the initial peak of the coronavirus pandemic, our conclusions are limited regarding the precise mechanism of the abduction paresis.

Treatment with azithromycin and hydroxychloroquine for 5 days per institutional protocol coincided with patient improvement and symptom cessation. 


\section{Conclusion}

Here we describe a case of unilateral abducens paresis and resulting diplopia as the sole ocular abnormality in a generally healthy male with mild COVID-19. As cases continue to rise, physicians should be aware that patients with COVID-19 may present with cranial nerve involvement even in mild cases without typical respiratory symptoms.

\section{Abbreviations}

\section{COVID-19}

novel coronavirus disease

SARS-CoV

severe acute respiratory syndrome coronavirus

\section{SARS-CoV-2}

severe acute respiratory syndrome coronavirus 2

\section{RT-PCR}

reverse transcriptase-polymerase chain reaction

CoV

coronavirus

\section{Declarations}

\section{Ethics approval and consent to participate}

All study methods adhered to the tenets of the Declaration of Helsinki for research involving human subjects. Informed consent was obtained from all subjects participating in the study.

\section{Consent for publication}

Consent for publication was obtained.

\section{Availability of data and material}

The data in the current case report are available in the Emek Medical Center records.

\section{Competing interests}

The authors of this case report have no competing interests to disclose. 


\section{Funding}

No financial support was provided.

\section{Author's contributions}

GBD designed the report as well as analyzed, acquired and interpreted the patient data and drafter the manuscript for intellectual content. $\mathrm{OH}$ designed the report as well as analyzed, acquired and interpreted the patient data and drafter the manuscript for intellectual content. HS designed the report as well as analyzed, acquired and interpreted the patient data and drafter the manuscript for intellectual content. SB designed the report as well as analyzed, acquired and interpreted the patient data and drafter the manuscript for intellectual content. GD designed the report as well as analyzed, acquired and interpreted the patient data and drafter the manuscript for intellectual content. DB designed the report as well as analyzed, acquired and interpreted the patient data and drafter the manuscript for intellectual content. All authors read and approved the final manuscript.

\section{Acknowledgments}

The authors acknowledge the patient for his generous cooperation.

\section{References}

1. World Health Organization Coronavirus disease 2019 (COVID-19) dashboard (2020) Geneva, Switzerland. Available online: https://covid19.who.int/ Accessed 30 July 2020

2. Wu P, Duan F, Luo $C$ et al (2020) Characteristics of ocular findings of patients with coronavirus disease 2019 (COVID-19) in Hubei province, China. JAMA Ophthalmol 138(5):575-578. doi:10.1001/jamaophthalmol.2020.1291

3. Mao L, Jin H, Wang M et al (2020) Neurologic Manifestations of Hospitalized Patients with Coronavirus Disease 2019 in Wuhan, China. JAMA Neurol 77(6):1-9. doi:10.1001/jamaneurol.2020.1127

4. Elder C, Hainline C, Galetta SL et al (2016) Isolated abducens nerve palsy: update on evaluation and diagnosis. Curr Neurol Neurosci Rep 16(8):69. doi:10.1007/s11910-016-0671-4

5. Li YC, Bai WZ, Hashikawa T (2020) The neuroinvasive potential of SARS-CoV-2 may play a role in the respiratory failure of COVID-19. J Med Virol 92(6):552-555. doi:10.1002/jmv.25728

6. Merad M, Martin JC (2020) Pathological inflammation in patients with COVID-19: a key-role for monocytes and macrophages. Nat Rev Immunol 20(6):355-362. doi:10.1038/s41577-020-0331-4. 
Online ahead of print

7. Gutiérrez-Ortiz C, Méndez A, Rodrigo-Rey S et al (2020) Miller Fisher Syndrome and polyneuritis cranialis in COVID-19. Neurology. doi: 10.1212/WNL.0000000000009619. Online ahead of print

8. Dinkin M, Gao V, Kahan J et al (2020) COVID-19 presenting with ophthalmoparesis from cranial nerve palsy. Neurology. doi:10.1212/WNL.0000000000009700. Online ahead of print

9. Falcone MM, Rong AJ, Salazar $\mathrm{H}$ et al (2020) Acute abducens nerve palsy in a patient with the novel coronavirus disease (COVID-19). J AAPOS. S1091-8531(20)30116-6. doi:

10.1016/j.jaapos.2020.06.001. Online ahead of print

\section{Figures}
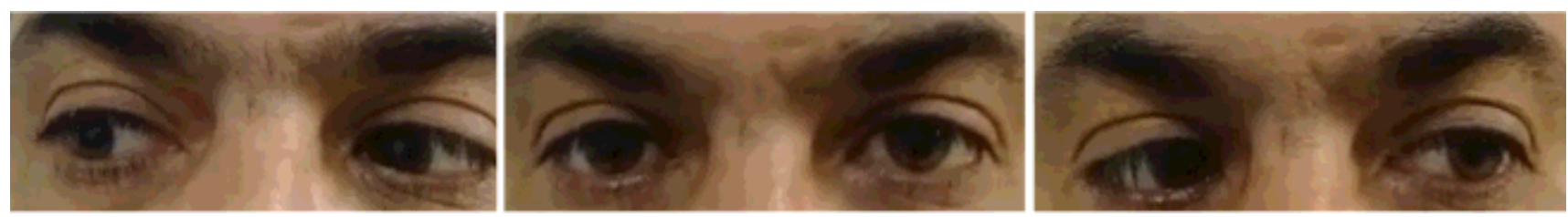

Figure 1

Horizontal Eye Movements Examination of extraocular horizontal eye movement revealed limitation to abduction in the left eye. Orthophoria was noted in right gaze. 